$16^{\text {th }}$ International Conference on

AEROSPACE SCIENCES \& AVIATION TECHNOLOGY,

ASAT - 16 - May 26 - 28, 2015, E-Mail: asat@ mtc.edu.eg

Military Technical College, Kobry Elkobbah, Cairo, Egypt

Tel : +(202) 24025292 - 24036138, Fax: +(202) 22621908

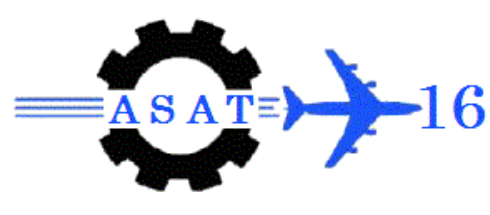

\title{
Analytical Formulas for Dipole Radiation Buried inside Planar Stratified Media
}

\author{
Samira Tadros Bishay ${ }^{*}$, Osama M. Abo-Seida ${ }^{\dagger}$, and Hanan Shehata Shoeib
}

\begin{abstract}
In this paper, analytical formulas have been derived for the electromagnetic fields (EMFs) radiated from a vertical magnetic dipole (VMD) buried in planar stratified media. Three planar-layered conducting media model are adopted: air, seawater and ground. Expressions for the Hertz vector which are used to determine the electric and magnetic field components of the dipole in the three planar-layered media are reduced to the integrals obtained by Sommerfeld previously. A simple technique with the aid of the complex image theory is used to obtain highly accurate analytical expressions for Sommerfeld integrals (SI) that arise in these calculations, which earlier were very tedious and complicated to evaluate. Closed-form expressions for the far field in the three regions due to a vertical magnetic dipole buried in second region (sea-water) are calculated and their physical meanings are discussed. The results are numerically evaluated and plotted. In addition, the results obtained in the three regions are compared with each other and with those mentioned elsewhere, and are proven that this method is very effective and simple. The formulas and computations can be applied to the communication in lower frequencies region. The achieved results will also be useful for remote sensing of the ocean surface, especially when the transmitter is close to the surface.
\end{abstract}

Keywords: Far-field, radiation in the sea, electromagnetic propagation, stratified media, buried vertical magnetic dipole (VMD), Sommerfeld Integral (SI).

\section{Introduction}

The electromagnetic wave radiated from a vertical magnetic dipole (VMD) buried or submerged in planar stratified media is of interest for remote sensing, communication purposes, radar, microwave systems and applied optics.

The problem of dipole radiation over media was originally formulated by Arnold Sommerfeld, in his classic work published in 1909 [1]. Since then, this problem has received a significant amount of attention and hundreds of papers have been published on the subject. The complete history of the problem is beyond the scope of this work, but suffices to say that independent derivations by Weyl [2], Norton [3] and others have presented more accurate representations for the field of the dipole, and have confirmed that there is an error in the sign of Sommerfeld's paper [1]. In 1941, Norton [4] has developed his formula for the field components to a stage where numerical results can readily be obtained. :

* Corresponding Author: Prof. Dr. Samira T. Bishay, Mathematics Department, Faculty of Science, Ain Shams University, Cairo, Egypt; stbishay@yahoo.com

$\dagger \quad$ Faculty of Science, Kafr El-Sheikh University, Egypt; aboseida@ sci.kfs.edu.eg

* Canal High Institute of Engineering \& Technology, Suez, hanan.shehata@ yahoo.com, 
In the pioneering work by Wait [5 -9],the Sommerfeld integral (SI) for the electromagnetic field of electric and magnetic dipoles radiating in the stratified medium (layered region) were evaluated by using asymptotic methods, contour integration, and branch cuts.In 1966,Baños [10] represented a complete perspective of the historical development of the mathematical analysis of the problem.

The electromagnetic fields of vertical and horizontal electric dipoles on or near the boundary between two different media have been well known in terms of closed-form expressions for many years. When this classical problem is solved, the Sommerfeld integral will occur and there is no way of avoiding the evaluation of the Sommerfeld integral for either numerical or closed form solutions. Due to the highly oscillatory and slowly decay nature of the Sommerfeld integral, it was difficult to apply a direct numerical integration technique to evaluate these integrals. Therefore, some well-known approaches such as steepest saddlepoint method, branch cut method and stationary phase point method, have been employed to obtain some approximate solutions. However, these methods, involving lengthy algebra and several transformations, are very tedious and complicated. Moreover, the properties of the electromagnetic field radiated by a dipole get more complicated when the boundary includes a layer of third material with intermediate properties.

In the early 1980's, Chew and Kong [11] computed the electromagnetic field of a horizontal dipole (HD) on a two-layer earth. The medium was assumed to be low-loss such that the image-source fields were important. Integral representations of image source fields were evaluated with uniform asymptotic approximations. In 1982, Bannister [12] derived expressions for the field components of a horizontal electric dipole (HED) in the presence of a conducting half-space and his formals were valid from the quasi-static to the far-field ranges. In a series of works by King [13 -15], the complete formulas have been obtained for the electromagnetic fields excited by horizontal electric dipole (HED) and vertical electric dipole (VED) in planar two- and three- layered media.

In 1988, Chew [16] was the first who derived simple and accurate formula (simple technique) for Sommerfeld integral. This formula was represented in an easily remembered steps. In 2001, Long et al. [17] developed the simple technique [16] and derived the far-field approximation of the Sommerfeld integral rapidly depending on the stationary phase-point. Furthermore, the slowly varying part in the Sommerfeld integral has no singularities at the stationary phase point, leading to use Chew's formulas in other similar problems to calculate the far-field.

The subsequent developments on the electromagnetic field of a dipole in layered region have been carried out by many investigators, especially including Long et al. technique [17]. Recently, many papers were published by Bishay et al. [18-21] that treated analytically the electromagnetic field of a vertical magnetic dipole in the presence of a three-layered rough region in details because of its many useful applications, especially in microstrip antenna.

In this research, in order to tip the scales, we substituted the rough region used previously in Bishay et al. [18-21] with a planar media. Therefore, to obtain higher degree of numerical efficiency, higher accuracy and faster results, we used in this paper Long et al. [17] technique with the aid of the complex image theory [12] to derive closed-form expressions for the far field in the three planar layered media due to a vertical magnetic dipole buried in second layered (sea water). The physical meaning of these expressions is discussed and the numerical computations are also carried out. In addition, the results are represented graphically and compared with those mentioned elsewhere.

\section{Geometric Description and Basic Formulation}

The relevant geometry that is illustrated in Fig.1, shows the model used in the calculation where it comprises three layers (air, sea, and ground). The source is a vertical magnetic dipole (VMD) located in the sea (the middle layer) at depth $d_{1}$ horizontally. Physically, this 
represents a small loop antenna of area $S_{0}$ carrying a circulating current $I . R$ is the distance between the source and an observing point $P(r, z)$ which is located in the sea at depth $d_{2}$. We suppose that the sea is of finite mean thickness $a$. If we describe, the geometry of the system by using the cylindrical polar coordinates $(r, \varphi, z)$, then for the whole space $0 \leq r<\infty$ and $0 \leq \varphi \leq 2 \pi$. Moreover, the air and the ground layers are infinite upward and downward along the $\mathrm{z}$-axis, respectively. All regions are also infinite sideward and are homogeneous. A fundamental assumption in this analysis is that the displacement current (in the sea) is negligible compared to the conduction current [20].

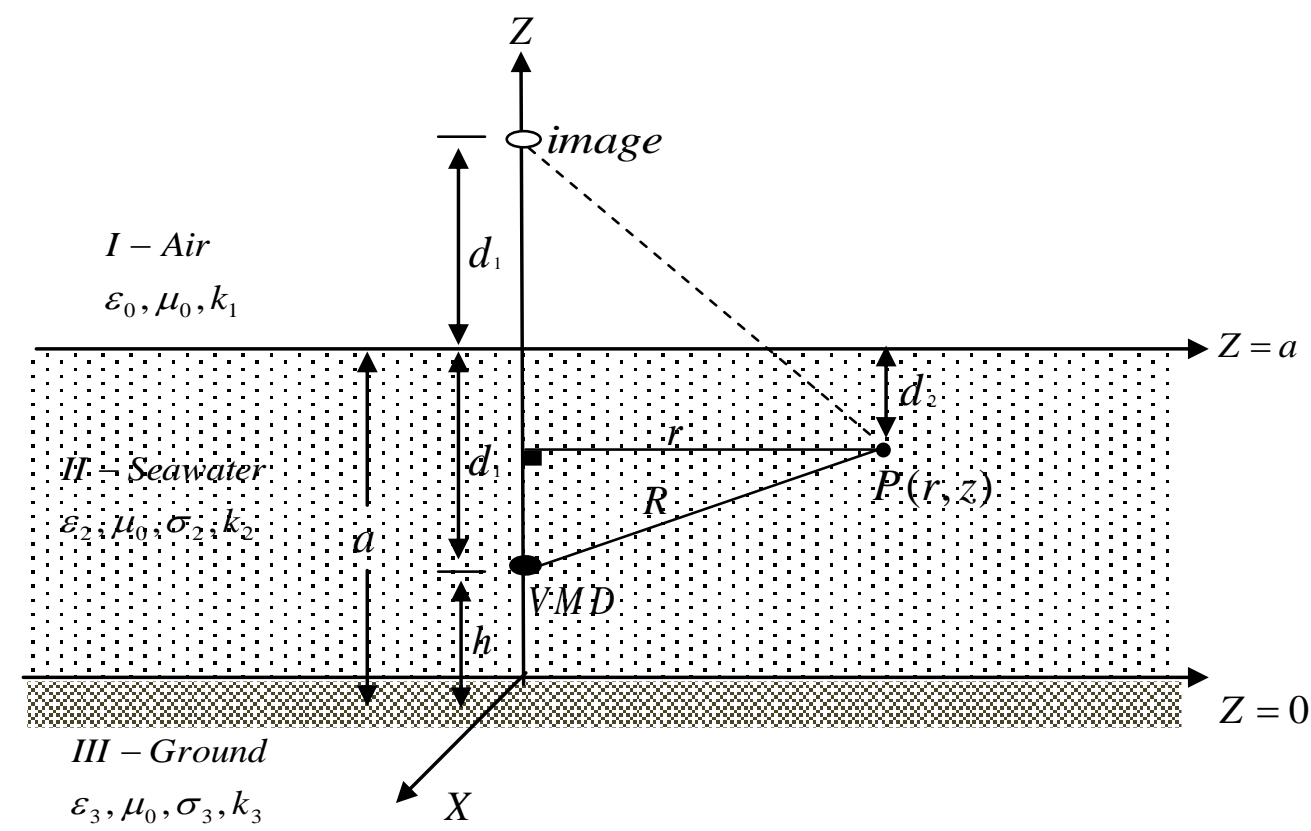

Fig. (1): Geometric configuration of a VMD located in the sea with a planar interfaces.

It is well known that the electromagnetic field generated by a vertical magnetic dipole (a small horizontal loop antenna) can be derived from the magnetic Hertz vector $\vec{\Pi}$ which has only one component $\Pi_{z}$ in the $z$-direction for the time variation $e^{i \omega t}$; consequently, the components of the electric and magnetic fields are given by [22]:

$$
E_{\varphi}=-i \omega \mu_{0} \frac{\partial \Pi_{Z}}{\partial r}, H_{r}=-\frac{\partial^{2} \Pi_{Z}}{\partial r \partial z} \text { and } H_{z}=-\left(\frac{\partial}{\partial r^{2}}+\frac{1}{r} \frac{\partial}{\partial r}\right) \Pi_{Z} .
$$

The tangent components of either electric or magnetic fields are continuous at the boundary, so the Hertz vector and its derivative by $\mathrm{z}$ are continuous [22]. Then, the $\mathrm{z}$-component of the Hertz vector $\Pi_{z}$ should satisfy the following boundary conditions:

$$
\begin{array}{llll}
\Pi_{Z}^{1}=\Pi_{Z}^{2} & , \quad \partial \Pi_{Z}^{1} / \partial z=\partial \Pi_{Z}^{2} / \partial z & \text { at } & \mathrm{z}=\mathrm{a} \\
\Pi_{Z}^{2}=\Pi_{Z}^{3} & , \quad \partial \Pi_{Z}^{2} / \partial z=\partial \Pi_{Z}^{3} / \partial z & \text { at } & \mathrm{z}=0
\end{array}
$$

Note that, the Sommerfeld radiation conditions [23] in the first and third media should be satisfied for $\left(r^{2}+z^{2}\right)^{1 / 2} \rightarrow \infty$. 


\section{The Integral Representation of the Fields in Three Media}

According to Sommerfeld [23], the z-component of the Hertz vector in each region at the general observation point $P(r, z)$ can be obtained as follows:

$$
\begin{array}{cr}
\Pi_{z}^{1}(r, z)=\frac{I S_{0}}{8 \pi} \int_{-\infty}^{\infty} A(\lambda) e^{-u_{1} z} J_{0}(\lambda r) d \lambda & \text { at } \quad a \leq z<\infty \\
\Pi_{z}^{2}(r, z)=\frac{I S_{0}}{8 \pi} \int_{-\infty}^{\infty}\left\{\frac{\lambda}{u_{2}} e^{-u_{2}|z-h|}+B(\lambda) e^{u_{2} z}+C(\lambda) e^{-u_{2} z}\right\} J_{0}(\lambda r) d \lambda & \text { at } \quad 0 \leq z \leq 0 \\
\Pi_{Z}^{3}(r, z)=\frac{I S_{0}}{8 \pi} \int_{-\infty}^{\infty} D(\lambda) e^{u_{3} z} J_{0}(\lambda r) d \lambda & \text { at } \quad-\infty<z \leq 0
\end{array}
$$

where $u_{i}=\sqrt{\lambda^{2}-k_{i}^{2}},(i=1,2,3)$ and its real part is positive, and $J_{0}(\lambda r)$ is the Bessel function of order zero. $A(\lambda), B(\lambda), C(\lambda)$, and $D(\lambda)$ are the constant functions, which we find them by combining the boundary conditions (2) and (3) leading to:

$$
\begin{gathered}
A(\lambda)=\frac{2 \lambda e^{u_{1} a}}{u_{21}^{+}}\left(\frac{e^{-u_{2}(a-h)}+r_{23} e^{-u_{2}(a+h)}}{1-r_{21} r_{23} e^{-2 u_{2} a}}\right), \\
B(\lambda)=\frac{\lambda e^{u_{2} a}}{u_{2}}\left(\frac{r_{21} e^{-u_{2}(a-h)}+r_{21} r_{23} e^{-u_{2}(a+h)}}{1-r_{21} r_{23} e^{-2 u_{2} a}}\right), \\
C(\lambda)=\frac{\lambda}{u_{2}}\left(\frac{r_{23} e^{-u_{2} h}+r_{21} r_{23} e^{-u_{2}(2 a-h)}}{1-r_{21} r_{23} e^{-2 u_{2} a}}\right) \\
D(\lambda)=\frac{2 \lambda}{u_{23}^{-}}\left(\frac{r_{23} e^{-u_{2} h}+r_{21} r_{23} e^{-u_{2}(2 a-h)}}{1-r_{21} r_{23} e^{-2 u_{2} a}}\right)
\end{gathered}
$$

where $r_{i j}=u_{i j}^{-} / u_{i j}^{+}=u_{i}-u_{j} / u_{i}+u_{j},(i, j=1,2,3)$ is the Fresnel reflection coefficient.

The non-vanishing components of the electric and magnetic fields in the three regions can be directly obtained after a fair amount of manipulative algebra, by substituting from (3) in (1) as follows:

In the air region at $a \leq z<\infty$ :

$$
\begin{gathered}
E_{\varphi}^{1}(r, z)=\frac{-i \omega \mu_{0} I S_{0}}{8 \pi} \int_{-\infty}^{\infty} \lambda A(\lambda) e^{-u_{1} z} H_{1}^{(2)}(\lambda r) d \lambda, \\
H_{r}^{1}(r, z)=\frac{I S_{0}}{8 \pi} \int_{-\infty}^{\infty} \lambda u_{1} A(\lambda) e^{-u_{1} z} H_{1}^{(2)}(\lambda r) d \lambda, \\
H_{z}^{1}(r, z)=\frac{I S_{0}}{8 \pi} \int_{-\infty}^{\infty} \lambda^{2} A(\lambda) e^{-u_{1} z} H_{0}^{(2)}(\lambda r) d \lambda .
\end{gathered}
$$


In the seawater region at $\mathrm{O} \leq z \leq a$ :

$$
\begin{gathered}
E_{\varphi}^{2}(r, z)=\frac{-i \omega \mu_{o} I S_{o}}{8 \pi} \int_{-\infty}^{\infty}\left(\frac{\lambda}{u_{2}} e^{-u_{2}|z-h|}+B(\lambda) e^{u_{2} z}+C(\lambda) e^{-u_{2} z}\right) \lambda H_{1}^{(2)}(\lambda r) d \lambda, \\
H_{r}^{2}(r, z)=\frac{-I S_{o}}{8 \pi} \int_{-\infty}^{\infty}\left(-\frac{\lambda}{u_{2}} e^{-u_{2}|z-h|}+B(\lambda) e^{u_{2} z}-C(\lambda) e^{-u_{2} z}\right) u_{2} \lambda H_{1}^{(2)}(\lambda r) d \lambda \\
H_{z}^{2}(r, z)=\frac{I S_{o}}{8 \pi} \int_{-\infty}^{\infty}\left(\frac{\lambda}{u_{2}} e^{-u_{2}|z-h|}+B(\lambda) e^{u_{2} z}+C(\lambda) e^{-u_{2} z}\right) \lambda^{2} H_{0}^{(2)}(\lambda r) d \lambda,
\end{gathered}
$$

In the ground region at $-\infty<z \leq 0$ :

$$
\begin{gathered}
E_{\varphi}^{3}(r, z)=\frac{-i \omega \mu_{0} I S_{0}}{8 \pi} \int_{-\infty}^{\infty} \lambda D(\lambda) e^{u_{3} z} H_{1}^{(2)}(\lambda r) d \lambda, \\
H_{r}^{3}(r, z)=\frac{-I S_{0}}{8 \pi} \int_{-\infty}^{\infty} \lambda u_{3} D(\lambda) e^{u_{3} z} H_{1}^{(2)}(\lambda r) d \lambda, \\
H_{z}^{3}(r, z)=\frac{I S_{0}}{8 \pi} \int_{-\infty}^{\infty} \lambda^{2} D(\lambda) e^{u_{3} z} H_{0}^{(2)}(\lambda r) d \lambda .
\end{gathered}
$$

It should be noted that, the following notations are introduced:

$$
\begin{gathered}
J_{1}(\lambda r)=-\lambda \frac{\partial J_{0}(\lambda r)}{\partial r}, \quad\left(\frac{\partial^{2}}{\partial r^{2}}+\frac{1}{r} \frac{\partial}{\partial r}\right) J_{0}(\lambda r)=-\lambda^{2} J_{0}(\lambda r) \\
J_{0}(x)=\frac{1}{2}\left\{H_{0}^{(1)}(x)+H_{0}^{(2)}(x)\right\} \quad, \quad H_{0}^{(1)}(-x)=-H_{0}^{(2)}(x)
\end{gathered}
$$

where $H_{o}^{(2)}(\lambda r)$ and $H_{1}^{(2)}(\lambda r)$ are the second kind Hankel function of order zero and one, respectively.

\section{Analytical Solution for the Far-Field in Air}

In this section, we will discuss the process of solution for the far-field in the air region. In this case the observing point $P(r, z)$ is at distance $d_{2}$ over the sea surface. We focus our attention on (5a) to calculate the electric field $E_{\varphi}^{1}$. The magnetic fields $H_{r}^{1}$ and $H_{z}^{1}$ can be also calculated in a similar way. It is clear that, the integral (5a) represents the upward wave from the sea surface to the observing point. Substituting by the value of the constant function $A(\lambda)$ from (4a) in (5a), the electric field $E_{\varphi}^{1}$ can be rewritten as follows:

$$
E_{\varphi}^{1}=\frac{-i \omega \mu_{0} I S_{0}}{8 \pi} \int_{-\infty}^{\infty} \frac{2 \lambda}{u_{21}^{+}} e^{-u_{1} d_{2}-u_{2} d_{1}}\left(\frac{1+r_{23} e^{-2 u_{2} h}}{1-r_{21} r_{23} e^{-2 u_{2} a}}\right) H_{1}^{(2)}(\lambda r) \lambda d \lambda .
$$

Taking into account the following relations:

$$
\left|r_{21} r_{23} e^{-2 u_{2} a}\right| \prec \prec 1 \quad \text { and } \quad \frac{1}{1-x}=\sum_{m=0}^{\infty} x^{m}
$$


then the denominator in (10) can be expressed as an infinite series:

$$
\begin{aligned}
E_{\varphi}^{1}=\frac{-i \omega \mu_{0} I S_{0}}{8 \pi} \int_{-\infty}^{\infty} \frac{2 \lambda}{u_{21}^{+}} e^{-u_{1} d_{2}-u_{2} d_{1}}\left(1+r_{23} e^{-2 u_{2} h}\right) \times \\
{\left[\sum_{m=0}^{\infty}\left(r_{21} r_{23} e^{-2 u_{2} a}\right)^{m}\right] H_{1}^{(2)}(\lambda r) \lambda d \lambda }
\end{aligned}
$$

According to the complex image theory [12], when the condition $\left|k_{2}^{2}\right| \succ \succ\left|k_{1}^{2}\right|$ is satisfied, we have:

$$
r_{21}=u_{21}^{-} / u_{21}^{+} \approx e^{-u_{1} d} \quad, \quad 2 u_{1} / u_{21}^{+} \approx 1-e^{-u_{1} d}
$$

where $d=-2 i / k_{2}$. Using the formulas (13) and properly arranging (12), we have:

$$
E_{\varphi}^{1}=\frac{-i \omega \mu_{0} I S_{0}}{8 \pi} \sum_{m=0}^{\infty}\left(\sum_{i=1}^{4} \Phi_{i}\right)
$$

where

$$
\begin{gathered}
\Phi_{1}=\int_{-\infty}^{\infty} \frac{\lambda}{u_{1}}\left(r_{23}\right)^{m} e^{-u_{1}\left(d_{2}+m d\right)-u_{2}\left(d_{1}+2 m a\right)} H_{1}^{(2)}(\lambda r) \lambda d \lambda, \\
\Phi_{2}=\int_{-\infty}^{\infty} \frac{\lambda}{u_{1}}\left(r_{23}\right)^{m+1} e^{-u_{1}\left(d_{2}+m d\right)-u_{2}\left(d_{1}+2 m a+2 h\right)} H_{1}^{(2)}(\lambda r) \lambda d \lambda \\
\Phi_{3}=-\int_{-\infty}^{\infty} \frac{\lambda}{u_{1}}\left(r_{23}\right)^{m} e^{-u_{1}\left(d_{2}+(m+1) d\right)-u_{2}\left(d_{1}+2 m a\right)} H_{1}^{(2)}(\lambda r) \lambda d \lambda \\
\Phi_{4}=-\int_{-\infty}^{\infty} \frac{\lambda}{u_{1}}\left(r_{23}\right)^{m+1} e^{-u_{1}\left(d_{2}+(m+1) d\right)-u_{2}\left(d_{1}+2 m a+2 h\right)} H_{1}^{(2)}(\lambda r) \lambda d \lambda .
\end{gathered}
$$

To find the value of $\Phi_{1}$, we used the technique in [17]. Therefore, the integral (15a) can be rewritten as:

$$
\Phi_{1}=\int_{-\infty}^{\infty}\left[\frac{\lambda}{u_{1}} e^{-u_{1}\left(d_{2}+m d\right)} H_{0}^{(2)}(\lambda r)\right]\left[\left(r_{23}\right)^{m} e^{-u_{2}\left(d_{1}+2 m a\right)} \frac{H_{1}^{(2)}(\lambda r)}{H_{0}^{(2)}(\lambda r)} \lambda\right] d \lambda .
$$

when $\lambda r \rightarrow \infty$, the first factor in (16) is rapidly varying while the second one is slowly varying. Moreover, according to Jones [22], the location of the stationary phase point is given by:

$$
\frac{\partial}{\partial \lambda} i\left[-\left(\sqrt{k_{1}^{2}-\lambda^{2}}\right)\left(d_{2}+m d\right)-\lambda r\right]_{\lambda=\lambda_{1}}=0
$$

which has the following solution:

$$
\lambda_{1}=r k_{1} / \bar{R}_{m} \quad, \bar{R}_{m}=\sqrt{r^{2}+\left(d_{2}+m d\right)^{2}}
$$

When $\lambda r \rightarrow \infty$, we can use the following approximation for Hankel function: 


$$
H_{m}^{(2)}(\lambda r) \approx \sqrt{\frac{2}{\pi \lambda r}} e^{-i(\lambda r-m \pi / 2-\pi / 4)}
$$

After using (19) and some straight forward manipulations, Eq. (16) can be expressed as:

$$
\Phi_{1}=\frac{2 i r k_{1}}{\bar{R}_{m}^{2}}\left(\frac{1-n}{1+n}\right)^{m} e^{-i k_{1} \bar{R}_{m}-i k_{2}\left(d_{1}+2 m a\right)}
$$

By the same way, we can obtain:

$$
\begin{gathered}
\Phi_{2}=\frac{2 i r k_{1}}{\bar{R}_{m}^{2}}\left(\frac{1-n}{1+n}\right)^{m+1} e^{-i k_{1} \bar{R}_{m}-i k_{2}\left(d_{1}+2 m a+2 h\right)} \\
\Phi_{3}=\frac{-2 i r k_{1}}{\bar{R}_{m+1}^{2}}\left(\frac{1-n}{1+n}\right)^{m} e^{-i k_{1} \bar{R}_{m+1}-i k_{2}\left(d_{1}+2 m a\right)} \\
\Phi_{4}=\frac{-2 i r k_{1}}{\bar{R}_{m+1}^{2}}\left(\frac{1-n}{1+n}\right)^{m+1} e^{-i k_{1} \bar{R}_{m+1}-i k_{2}\left(d_{1}+2 m a+2 h\right)}
\end{gathered}
$$

where $n=k_{3} / k_{2}, \quad \bar{R}_{m+1}=\sqrt{r^{2}+\left(d_{2}+(m+1) d\right)^{2}}$ and the approximations $\boldsymbol{u}_{2} \approx i k_{2}$ and $u_{3} \approx i k_{3}$ are used because the condition $\left|k_{2}^{2}\right| \succ \succ 80\left|k_{1}^{2}\right|$ is satisfied [12].

Substituting by the values of $\Phi_{i}(i=1, \ldots 4)$ from (20) - (23) into (14), the final expression of $E_{\varphi}^{1}$ can be obtained as follows:

$$
E_{\varphi}^{1}=\frac{\omega \mu_{0} I S_{0}}{4 \pi} r k_{1} N e^{-i k_{2} d_{1}} \sum_{m=0}^{\infty}\left(\frac{1-n}{1+n}\right)^{m+1} e^{-i k_{2}(2 m a)}\left(\frac{e^{-i k_{1} \bar{R}_{m}}}{\bar{R}_{m}^{2}}-\frac{e^{-i k_{1} \bar{R}_{m+1}}}{\bar{R}_{m+1}^{2}}\right)
$$

where $N=\left\{(1-n / 1+n)+e^{-i k_{2}(2 h)}\right\}$. One of the contributions of this paper is equation (24), where the component of the electric filed that used to be calculated previously by Long et al. [17] with an approximation for $\lambda \approx k_{1}$ and $\bar{R}_{m} \approx \bar{R}_{m+1} \approx \bar{R}_{0}$ now we attain its value without this approximation.

The physical meaning of Eq. (24) can be explained that the first term indicates a series of waves, which propagate upward from the source and make $m$ round trips between the sea surface and the bottom, then travel along the path $\bar{R}_{m}$ in air and arrive at the field point, where $(1-n) /(1+n)$ is the reflection coefficient at the sea bottom. The reflection coefficient at the sea surface is regarded as almost unity. The second term indicates another series of waves, which propagate downward from the source first, then reflect upward at the sea bottom, and travel along the path $\bar{R}_{m+1}$ in air and arrive at the field point.

\section{Analytical Solution for the Far-Field in Sea-Water}

The field point $P(r, z)$ is at distance $d_{2}$ under the sea surface as illustrated in Fig.1. In order to evaluate the value of the field in the sea-water region, we must solve the integral equations (6). It is clear that the first term of the integral is the direct wave (the primary field) from the source to the observing point. This term is important, if the transmitter and receiver are set very closely. However, if the two points are separated far from each other, this term will vanish because the propagation path is in the sea and the attenuation is very large. The second term is concerned with $e^{u_{2} z}$, which represents the downward wave from the surface of the 
sea to the observing point. The last term, which is the third term is concerned with $e^{-u_{2} z}$, that represents the upward wave from the sea bottom to the observing point, if the receiver is close to the bottom, this term seems to be useful.

Starting with the integral equation (6a) and after substituting by the values of the constant functions $B(\lambda)$ and $C(\lambda)$ from (6b) and (6c), we can write the electric field in sea-water $E_{\varphi}^{2}$ after a fair amount of manipulative algebra as:

$$
E_{\varphi}^{2}(r, z)=\frac{-i \omega \mu_{o} I S_{o}}{8 \pi} \sum_{i=1}^{3} \Psi_{i},
$$

where

$$
\begin{gathered}
\Psi_{1}=\int_{-\infty}^{\infty} \frac{\lambda}{u_{2}} e^{-u_{2}|z-h|} H_{1}^{(2)}(\lambda r) \lambda d \lambda \\
\Psi_{2}=\int_{-\infty}^{\infty} \frac{\lambda}{u_{2}} e^{-u_{2}\left(d_{1}+d_{2}\right)} r_{21}\left(\frac{1+r_{23} e^{-2 u_{2} h}}{1-r_{21} r_{23} e^{-2 u_{2} a}}\right) H_{1}^{(2)}(\lambda r) \lambda d \lambda \\
\Psi_{3}=\int_{-\infty}^{\infty} \frac{\lambda}{u_{2}} e^{-u_{2}(z+h)} r_{23}\left(\frac{1+r_{21} e^{-2 u_{2} d_{1}}}{1-r_{21} r_{23} e^{-2 u_{2} a}}\right) H_{1}^{(2)}(\lambda r) \lambda d \lambda
\end{gathered}
$$

Starting with the integral Eq. (26a), and by using the same method in [17], we can rewrite $\Psi_{1}$ as:

$$
\Psi_{1}=\int_{-\infty}^{\infty}\left[\frac{\lambda}{u_{2}} e^{-u_{2}|z-h|} H_{0}^{(2)}(\lambda r)\right]\left[\lambda \frac{H_{1}^{(2)}(\lambda r)}{H_{0}^{(2)}(\lambda r)}\right] d \lambda
$$

When $\lambda r \rightarrow \infty$, the first factor in (27) is the rapidly varying part while the second one is the slowly varying part. Then, the location of the stationary phase point is given by:

$$
\frac{\partial}{\partial \lambda} i\left[-\left(\sqrt{k_{2}^{2}-\lambda^{2}}\right)(z-h)-\lambda r\right]_{\lambda=\lambda_{2}}=0
$$

The solution of equation (28) is:

$$
\lambda_{2}=r k_{2} / R \quad \text { and } \quad R=\sqrt{r^{2}+(z-h)^{2}}
$$

After solving the rapidly and slowly parts, we have the solution of $\Psi_{1}$ as follows:

$$
\Psi_{1}=2 i\left(\frac{r k_{2}}{R^{2}} e^{-i k_{2} R}\right)
$$

In the same manner, we obtain the solution of $\Psi_{2}$ and $\Psi_{3}$ as:

$$
\begin{gathered}
\Psi_{2}=\frac{4 k_{1}^{2} r}{k_{2}^{2}} N \sum_{m=0}^{\infty}(m+1)\left(\frac{1-n}{1+n}\right)^{m+1} \frac{e^{-i k_{1} R_{m+1}}}{R_{m+1}^{3}} e^{-i k_{2}\left(d_{1}+d_{2}+2 m a\right)} \\
\Psi_{3}=\frac{4 k_{1}^{2} r}{k_{2}^{2}} \sum_{m=0}^{\infty}\left(\frac{1-n}{1+n}\right)^{m+1} e^{-i k_{2}(z+h+2 m a)}\left(\frac{m e^{-i k_{1} R_{m}}}{R_{m}^{3}}+\frac{(m+1) e^{-i k_{1} R_{m+1}}}{R_{m+1}^{3}} e^{-2 i k_{2} d_{1}}\right)
\end{gathered}
$$


where $R_{m}=\sqrt{r^{2}+\{m d\}^{2}}$ and $R_{m+1}=\sqrt{r^{2}+\{(m+1) d\}^{2}}$.

From (30) in (25), it is easy to get the solution formula for the electric field $E_{\varphi}^{2}$ in sea-water as follows:

$$
\begin{aligned}
& E_{\varphi}^{2}=\frac{-i \omega \mu_{0} I S_{0} k_{1}^{2} r}{2 \pi k_{2}^{2}}\left(\frac{i k_{2}^{3}}{k_{1}^{2} R^{2}} e^{-i k_{2} R}+\sum_{m=0}^{\infty}\left(\frac{1-n}{1+n}\right)^{m+1} e^{-i k_{2}(2 m a)} \times\right. \\
& \left.\left\{\left(\frac{m e^{-i k_{1} R_{m}}}{R_{m}^{3}} e^{-i k_{2}(z+h)}\right)+\left((m+1) \frac{e^{-i k_{1} R_{m+1}}}{R_{m+1}^{3}}\right)\left(N e^{-i k_{2}\left(d_{1}+d_{2}\right)}+e^{-i k_{2}\left(z+h+2 d_{1}\right)}\right)\right\}\right)
\end{aligned}
$$

The physical meaning of (31) can be explained that the second term represents a series of waves, which propagate upward from the source and make $m$ round trips between the sea surface and the bottom, then travel along the path $R_{m}$ and arrive at the field point in seawater. The third term represents another series of waves, which propagate downward from the source first, then reflect upward at the sea bottom, and travel along the path $R_{m+1}$ and arrive at the field point.

\section{Analytical Solution for the Far-Field in The Ground}

In this section we will analyze the integral equations (7) to find the solution of the far field in the ground region (third-layered). In this case, the field point $P(r, z)$ is at distance $d_{2}$ under the sea surface. We begin with the integral equation (7a) and after substituting the value of the constant function $D(\lambda)$ from $(4 \mathrm{~d})$, the electric field $E_{\varphi}^{3}$ can be expressed as:

$$
E_{\varphi}^{3}=\frac{-i \omega \mu_{0} I S_{0}}{8 \pi} \int_{-\infty}^{\infty} \frac{2 \lambda}{u_{23}^{+}} e^{-u_{2} h-u_{3} z}\left(\frac{1+r_{21} e^{-2 u_{2} d_{1}}}{1-r_{21} r_{23} e^{-2 u_{2} a}}\right) H_{1}^{(2)}(\lambda r) \lambda d \lambda
$$

Using the first formula of (11) and (13), then the electric field $E_{\varphi}^{3}$ can be rewritten as:

$$
\begin{aligned}
E_{\varphi}^{3}=\frac{-i \omega \mu_{0} I S_{0}}{4 \pi} \sum_{m=0}^{\infty} \int_{-\infty}^{\infty} & \frac{\lambda}{u_{23}^{+}} e^{-u_{3} z-u_{2}(h+2 m a)-u_{1} m d} \times \\
& \left(r_{23}\right)^{m}\left(1+e^{-2 u_{2} d_{1}-u_{1} d}\right) H_{1}^{(2)}(\lambda r) \lambda d \lambda
\end{aligned}
$$

Using the similar procedures for finding $E_{\varphi}^{1}$ and $E_{\varphi}^{2}$, the final result of the electric field after some lengthy but straightforward manipulations can also be given by:

$$
\begin{gathered}
E_{\varphi}^{3}=\frac{-i \omega \mu_{0} I S_{0} k_{1}^{2} r}{\pi k_{2}^{2}}\left(\frac{1}{1+n}\right) e^{i k_{3} z-i k_{2} h} \sum_{m=0}^{\infty}\left(\frac{1-n}{1+n}\right)^{m} \times \\
e^{-i k_{2}(2 m a)}\left(\frac{m e^{-i k_{1} R_{m}}}{R_{m}^{3}}+\frac{(m+1) e^{-i k_{2} R_{m+1}}}{R_{m+1}^{3}} e^{-i k_{2}\left(2 d_{1}\right)}\right),
\end{gathered}
$$


It is clear that, the first term of (34) represents a series of waves, which propagate upward from the source and make $m$ round trips between the sea surface and the bottom, then travel along the path $R_{m}$ and arrive at the field point in ground. The second term represents another series of waves, which propagate upward from the source first, then reflect downward at the sea surface, and travel along the path $R_{m+1}$ and arrive at the field point.

\section{Numerical Results}

We here present some graphs showing the electric fields which is computed in three regions for different values of $a$ (thickness of the sea). The source is a VMD located in sea at height $h$ above the sea bottom. In these Figures, the vertical scale is normalized by the factor $\left(\omega \mu_{0} I S_{0} / 4 \pi\right)$. To attain the numerical calculation, we ascertain that $\sum_{m=0}^{50}$ is a match for $\sum_{m=0}^{\infty}$ under the conditions shown in Figures (2) - (10).

Figures (2), (5), and (8) show clearly that the decrease in the sea thickness $a$ led to an increase in the electric field. Moreover, Figures (3), (6) and (9) show that an increase in the height of the source $h$, increases the electric. Figures (4), (7) and (10) show that the electric field increases when the vertical distance of the field point $d_{2}$ is increased until it reaches to a certain value then decreased. Furthermore, Figures (11) - (13) show that the electric field is strong in sea-water region, where the source and the filed point are in the same region. These results coincide with previous results obtained by using other methods. However, this method is much easier and delivers these results more quickly and thus save the time exhausted in previous tedious approaches. Therefore, this method which is another contribution of this paper, is very important as it saves the researcher's time in finding the accurate results of the problem in a very short time.

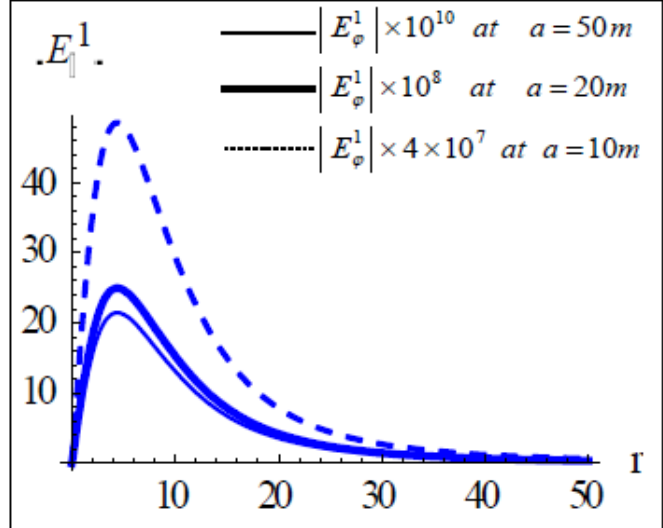

Figure (2): Variation of the absolute value of the electric field in air region $\left|E_{\varphi}^{1}\right|$ versus the radial distance (r) at $a=50,20,10 \mathrm{~m}$, respectively, $h=4 \mathrm{~m}$ and $d_{2}=5 \mathrm{~m}$.

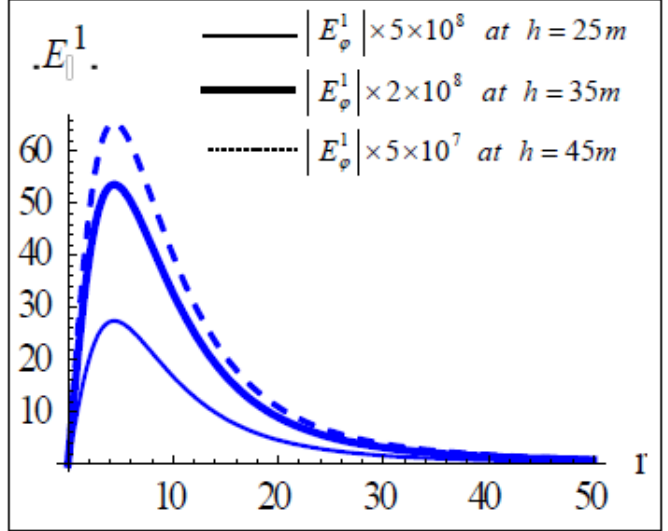

Figure (3): Variation of the absolute value of the electric field in air region $\left|E_{\varphi}^{1}\right|$ versus the radial distance ( $r$ ) at $a=50 \mathrm{~m}, h=25,35,45$ $m$, respectively, and $d_{2}=5 \mathrm{~m}$. 


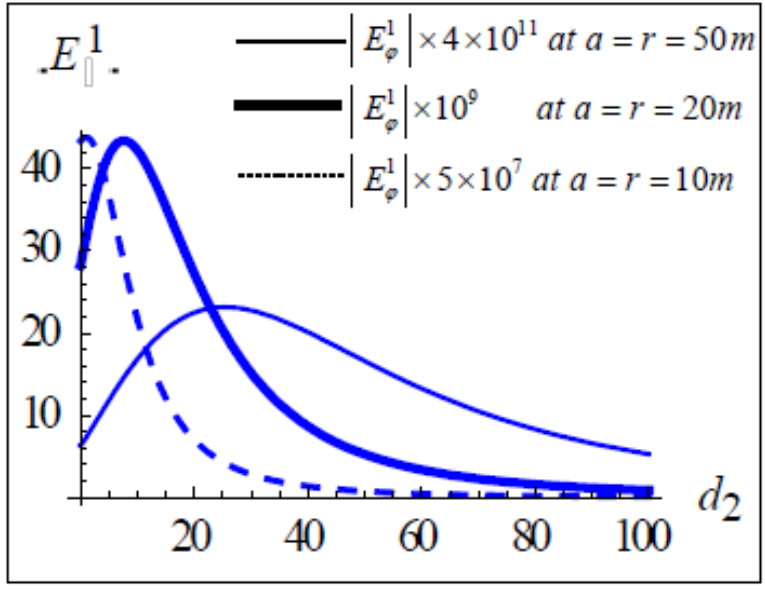

Figure (4): Variation of the absolute value of the electric field in air region $\left|E_{\varphi}^{1}\right|$ versus the vertical distance of the field point $d_{2}$ at $a=r=50,20,10 \mathrm{~m}$, respectively, and $h=4 \mathrm{~m}$.

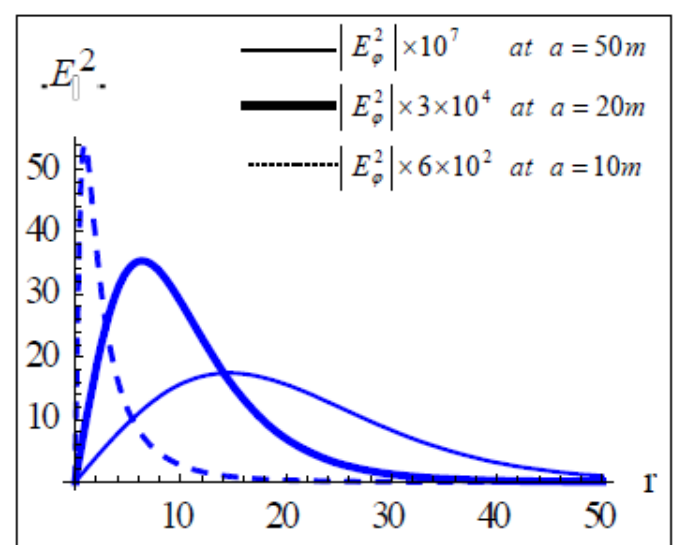

Figure (5): Variation of the absolute value of the electric field in seawater region $\left|E_{\rho}^{2}\right|$ versus the radial distance $(r)$ at $a=50,20$, $10 \mathrm{~m}$, respectively, $h=4 \mathrm{~m}$ and $d_{2}=5 \mathrm{~m}$.

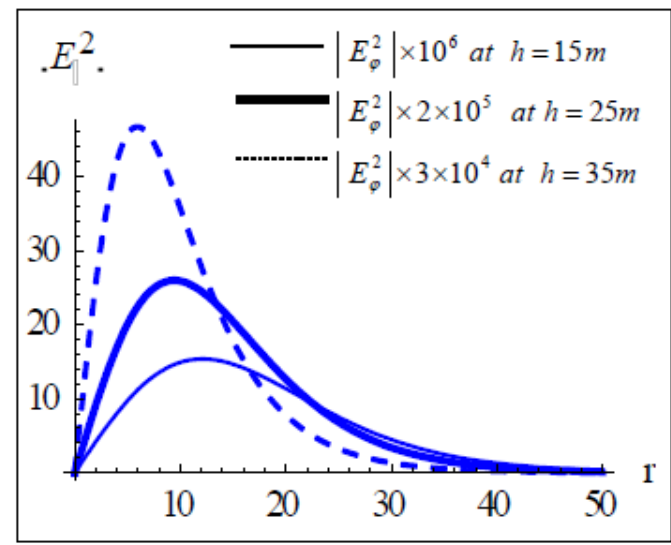

Figure (6): Variation of the absolute value of the electric field in seawater region $\left|E_{\varphi}^{2}\right|$ versus the radial distance (r) at $a=50 \mathrm{~m}$, $h=15,25,35 \mathrm{~m}$, respectively, and $d_{2}=5 \mathrm{~m}$. 


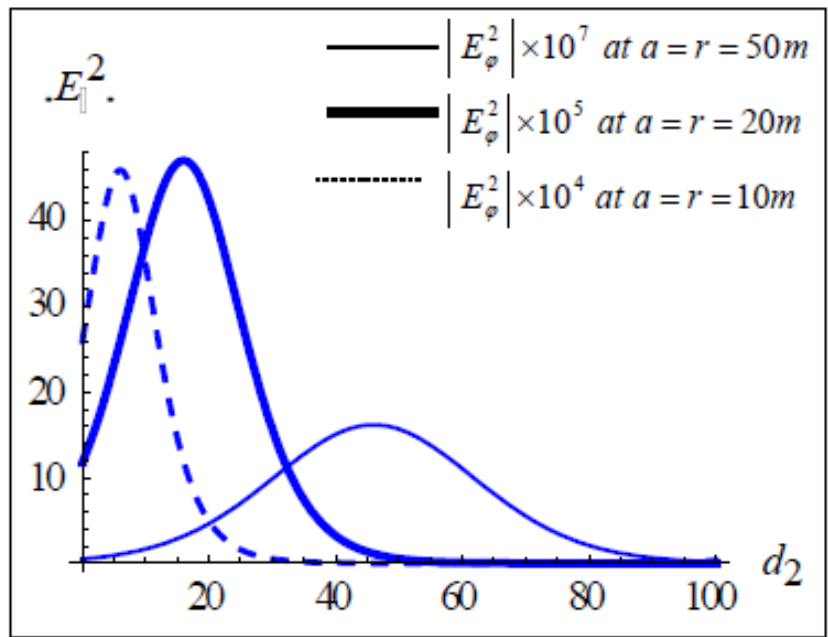

Figure (7): Variation of the absolute value of the electric field in seawater region $\left|E_{\varphi}^{2}\right|$ versus the vertical distance of the field point $d_{2}$ at $a=r=50,20,10 \mathrm{~m}$, respectively, and $h=4 \mathrm{~m}$.

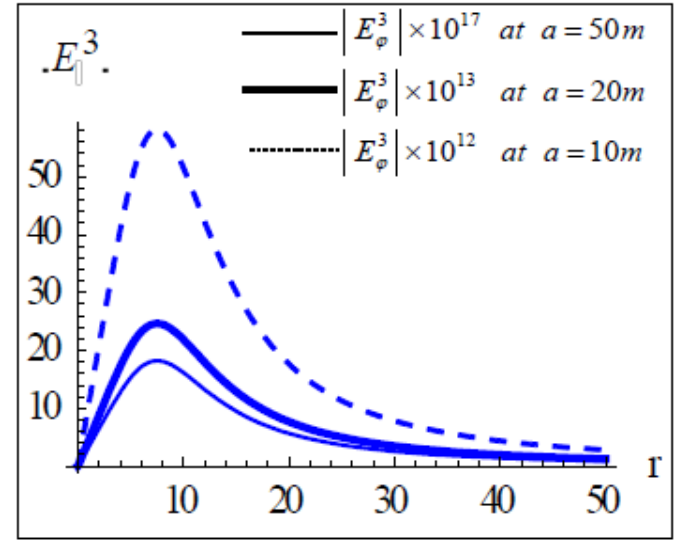

Figure 8): Variation of the absolute value of the electric field in ground region $\left|E_{\varphi}^{3}\right|$ versus the radial distance $(r)$ at $a=50,20,10 \mathrm{~m}$, $d_{2}=55,25,15 \mathrm{~m}$ respectively, and $h=4 \mathrm{~m}$.

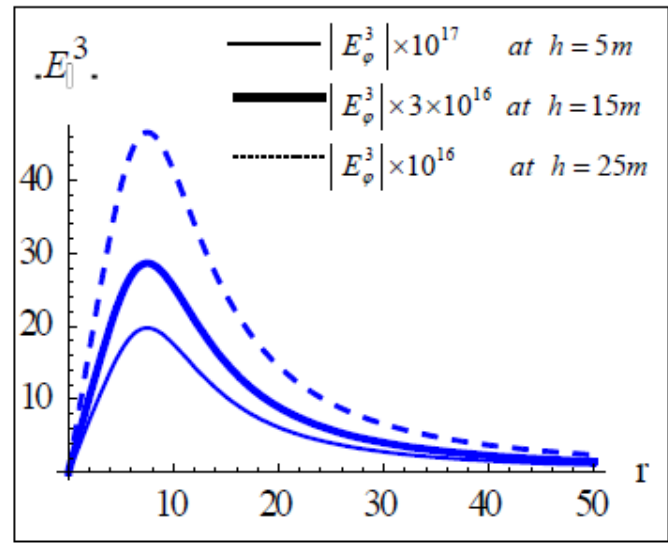

Figure (9): Variation of the absolute value of the electric field in ground region $\left|E_{\varphi}^{3}\right|$ versus the radial distance ( $r$ ) at $a=50 \mathrm{~m}, h=5,15$, $25 \mathrm{~m}$, respectively, and $d_{2}=55 \mathrm{~m}$. 


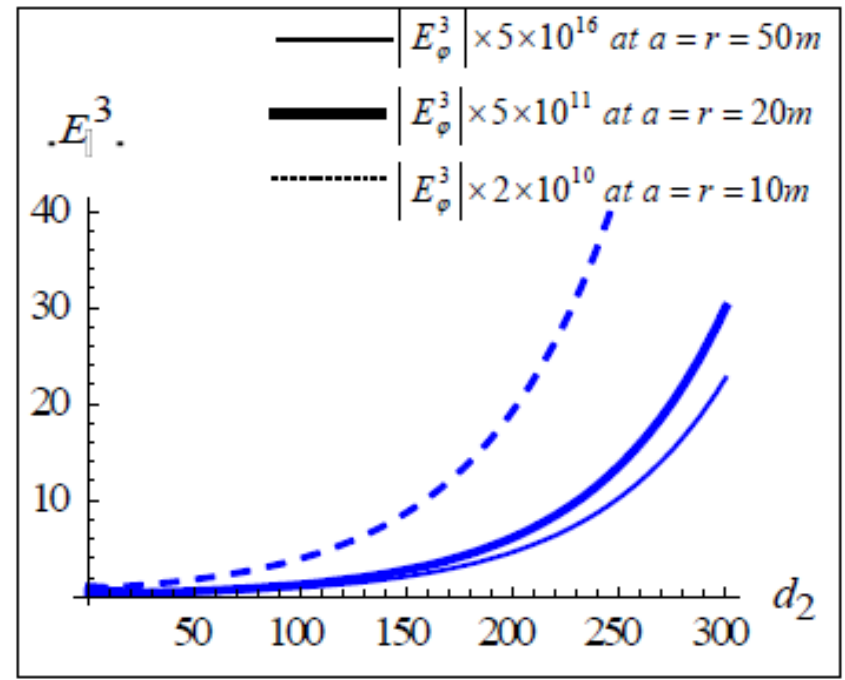

Figure (10): Variation of the absolute value of the electric field in ground region $\left|E_{\varphi}^{3}\right|$ versus the vertical distance of the field point $d_{2}$ at $a=r=50,20,10 \mathrm{~m}$, respectively, and $h=4 \mathrm{~m}$.

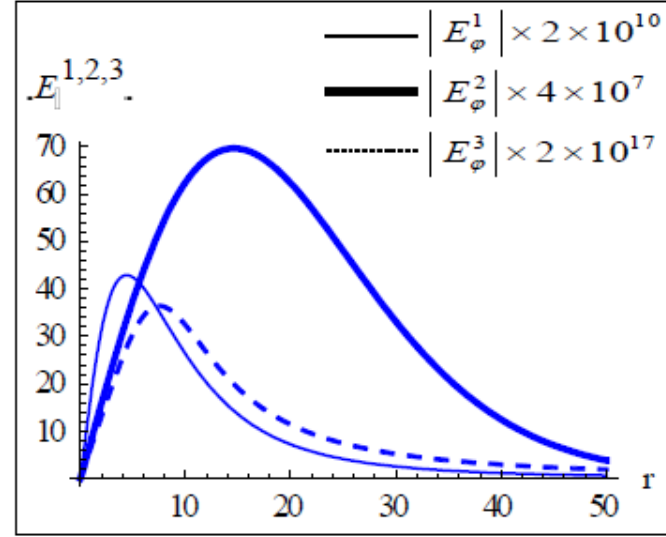

Figure (11): Variation of the absolute value of the electric field $\left|E_{\%}^{1,2,3}\right|$ in three regions: air, seawater and ground, respectively, versus the radial distance ( $r$ ) at $a=50 \mathrm{~m}$, $h=4 m$, and $d,=5,5,55 m$ respectively.

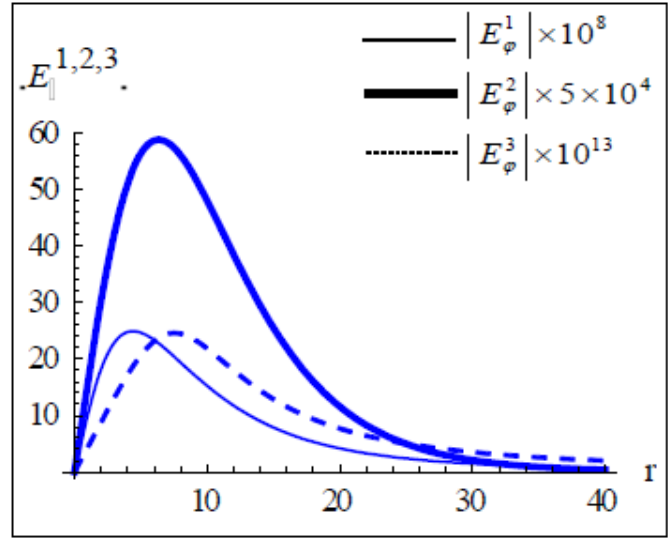

Figure (12): Variation of the absolute value of the electric field $\left|E_{\varphi}^{1,2,3}\right|$ in three regions: air, seawater and ground, respectively, versus the radial distance ( $r$ ) at $a=20 \mathrm{~m}$, $h=4 m$, and $d_{2}=5,5,25 m$ respectively. 


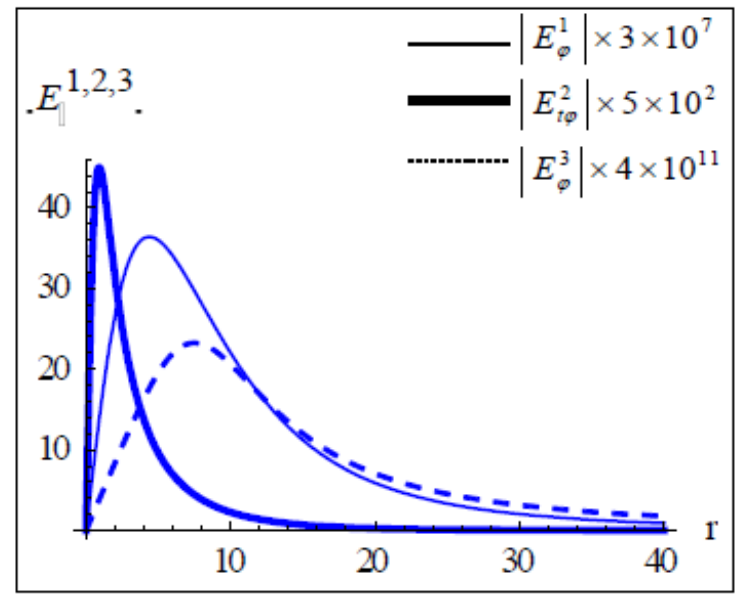

Figure (13): Variation of the absolute value of the electric field $\left|E_{\varphi}^{1,2,3}\right|$ in three regions: air, seawater and ground, respectively, versus the radial distance ( $r$ ) at $a=10 \mathrm{~m}, h=4 \mathrm{~m}$, and $d_{2}=5,5,15 \mathrm{~m}$ respectively.

\section{Discussion and Conclusion}

In this paper we summarized our research work and its visible deliverable are the complete formulas that have been derived for the electromagnetic fields generated by a vertical magnetic dipole embedded in sea (the three-layered media) with planar interfaces. The observation point is located in air, seawater, and ground, respectively. Moreover, the distinct physical meanings of the derived fields in each of the three regions are discussed separately. Numerical calculations of the field components are performed and illustrated.

This paper also contributes to the analysis of the EMFs which is based on this method, as it depends on the use of some integral identities and the identification of the stationary phase point of the integrand to evaluate SI rapidly. This was illustrated by showing the form solutions of the far-fields were first expanded, and then with the help of the complex image theory [12], closed form expressions of the far-field in the three regions were obtained simply. It is necessary to note that, the slowly varying part in SI has no singularities at the stationary phase point. The results of this work agreed to a great extent with the conventional stationary phase method or saddle -point method, but it is quicker and it can be easily used to calculate the far - fields for similar problems.

The results obtained are useful in many engineering applications such as underwater communication, mine communication, radar and geophysics exploring. It can also be used in developing fast and simple interpretation tools for estimating the earth structure and other remote sensing applications.

\section{References}

[1] Sommerfeld, "Über die Ausbreitung der Wellen in der drahtlosen Telegraphie," Ann. Physik, vol. 28, pp. 665-736, 1909; Vol. 81, pp. 1135-1153, 1926.

[2] H. Weyl, "Ausbreitung elektromagnetischer Wellen "uber einem ebenen Leiter," Ann. Physik, Vol. 60, pp. 481-500, 1919.

[3] K. A. Norton, "Propagation of radio waves over a plane earth", Nature, Vol. 135, pp. 954-955, June 1935.

[4] K. A. Norton, "The calculation of ground-wave field intensity over a finitely conducting spherical earth," Proc. Inst. of Radio Engrs., Vol. 29, No. 12, pp. 623 - 639, Dec. 1941. 
[5] J. R. Wait, "Radiation from a vertical electric dipole over a stratified ground," IRE Trans. Antennas Propagat., Vol. 1, No. 1, pp. 9-12, 1953.

[6] J. R. Wait, "Radiation from a vertical electric dipole over a stratified ground," IRE Trans. Antennas Propagat., Vol. 2, No. 4, pp. 144-146, 1954.

[7] J. R. Wait, "Radiation from a vertical electric dipole over a curved stratified ground," Journal of Res. Nat. Bur. Standards, Vol. 56, pp. 232-239, 1956.

[8] J. R. Wait, "Excitation of surface waves on conducting dielectric clad and corrugated surfaces," Journal of Res. Nat. Bur. Standards, Vol. 59, No. 6, pp. 365-377, 1957.

[9] J. R. Wait, "Electromagnetic Waves in Stratified Media," Oxford, England: Pergamon Press, New York, (1962) 1970.

[10] A. Baños, "Dipole Radiation in the Presence of a Conducting Half - space," Pergamon Press, Oxford, 1966.

[11] W. C. Chew and J. A. Kong, "Electromagnetic field of a dipole in a two-layer earth," Geophysics, Vol. 46, No. 3, pp. 309-315, Mar. 1981.

[12] P. R. Bannister, "The image theory of electromagnetic field of a horizontal electric dipole in the presence of a conducting half-space," Radio Sci., Vol. 17, No. 5, pp. 1095 1102, Sep.-Oct. 1982.

[13] R. W. P. King, "New formulas for the electromagnetic field of a vertical electric dipole in a dielectric or conducting half-space near its horizontal interface," J. Appl. Phys., Vol. 53, pp. 8476-8482, Dec. 1982; erratum, Vol. 56, pp. 3366, Dec. 1984.

[14] R. W. P. King, "The electromagnetic field of a horizontal electric dipole in the presence of a three-layered region," J. Appl. Phys., Vol. 69, No. 12, pp. 7987-7995, 1991.

[15] R. W. P. King, "The electromagnetic field of a horizontal electric dipole in the presence of a three-layered region: Supplement," J. Appl. Phys., Vol. 74, No. 8, pp. 4845-4548, 1993.

[16] W. C. Chew, "A quick way to approximate a Sommerfeld-Wely-Type integral," IEEE Trans. Antennas Propagat., Vol. 36, No. 11, pp. 1654-1657, Nov. 1988.

[17] Y. Long, H. Jiang and B. Rembold, "Far-Region Electromagnetic Radiation with a Vertical Magnetic Dipole in Sea," IEEE Trans. Antennas Propagat., Vol. 49, No. 6, pp. 992-996, June 2001.

[18] S. T. Bishay, O. M. Abo-Seida, and H. Sh. Shoeib, "Electromagnetic Radiation Fields in Three-Layered Media with rough Interfaces," IEEE Trans. Geosci. Remote Sensing, Vol. 50, No. 10, pp. 4006 - 4013, 2012.

[19] S. T. Bishay, O. M. Abo-Seida, and H. Sh. Shoeib, "Scattering from Layered-Structures with Rough Boundaries," Electromagnetics, Vol. 33, No. 7, pp. 491- 506 , 2013.

[20] S. T. Bishay, O. M. Abo-Seida, and H. Sh. Shoeib, "Wave Propagation in Air from a Vertical Magnetic Dipole Located in Three Rough-Layered Structures," Journal of Electromagnetic Waves and Applications (JEMWA), Vol. 27, No. 7, pp. 911 - 929, 2013.

[21] J. R. Wait, "Electromagnetic fields of sources in lossy media," in R. E. Collin and F. J. Zucker (Eds.). Antenna Theory. Part II. Chap. 24, pp. 438 - 514. McGraw-Hill. New York. 1969.

[22] D. S. Jones, "The Theory of Electromagnetism," Pregamon Press, London 1964.

[23] A. Sommerfeld, "Partial Differential Equations in Physics," London, U.K.: Academic Press, 1964.

[24] S. T. Bishay is with the Department of Mathematics, Faculty of Science, Ain Shams University, Cairo 11566, Egypt (e-mail: stbishay@yahoo.com).

[25] O. M. Abo-Seida is with the Department of Mathematics, Faculty of Science, Kafer ElSheikh University, Kafer El-Sheikh 33516, Egypt (e-mail: aboseida@ yahoo.com).

[26] H. Sh. Shoeib, is with the Department of Mathematics, Canal Institute of Engineering \& Technology, Suze, Egypt (e-mail: hanan.shehata@yahoo.com). 